\title{
Effect of the Superstructure Construction Method on the Cost and Duration of Bridge Projects in Sudan
}

\section{Hashim Mohamed Ahmed ${ }^{1}$}

\section{Eltayeb Hassan Onsa ${ }^{2}$}

1 Ministry of Planning and Infrastructures, Khartoum State.

E-mail: hashimsudan69@yahoo.com.

1 Faculty of Engineering Sciences, Omdurman Islamic University, E-mail: eonsah@gmail.com.

السودان بلدُّ واسعٌ يتكون من ستة عشر ولاية يوجد فيها آلاف الكيلومترات من الممرات

المائية والأودية ، جعلت الحاجة إلى تثبيد الجسور كبيرة لأجل تحسين الإتصال والتتمية. هناك عدة جسور تم تثبيدها في العقود الماضية في السودان من الخرسانة أو الفولاذ باستخدام عدة أنظمة من الجسور المعلقة والقوسية والجسور الجملونية والمستوية على كوابل وبطرق تتييد متعددة. إن اختيار أفضل طريقة لتشييد المنشأة العلوية للجسور تعتبر مهمة لنجاح مشاريع تشييد الجسور الذي يقاس عموماً بسرعة التشييد وقلة التكلفة المالية. تُلخص هذه الورقة المعلومات الرئيسة للجسور الكبيرة في السودان من حيث المعلومات الهندسية الأساسية وطريقة التتييد وزمن التشييد والتكلفة المالية وتقدم مقارنة بين طرق تثبيد الجسور الرئيسة بالسودان فيما يتعلق بتكلفة الجسر محسوبةً بالدولار الأمريكي لكل متر

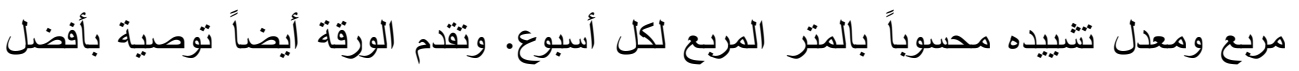
طرق لتشبيد المنشأة العلوية في الجسور بالسودان والتي ينوقع أن تساهم في تحسين تكلفة وتقليل مدة تشييد الجسور •

1 Ministry of Planning and Infrastructures, Khartoum State.

E-mail: hashimsudan69@yahoo.com.

2 Faculty of Engineering Sciences, Omdurman Islamic University, E-mail: eonsah@gmail.com. 


\begin{abstract}
Sudan, being a wide country consisting of sixteen states, in which thousands of kilometres of waterways and valleys are extended, bridges had become desperately needed for proper communication and development. There were many types of bridges in Sudan made of concrete, steel and composite sections showing various types of suspended, steel truss, arch and cable-stayed bridges which were constructed by different methods.

This paper summarizes the data of main bridges in Sudan; it shows the construction duration, cost, and type of the superstructure and presents comparison between bridges' superstructure construction method in terms of cost in US\$ per $\mathrm{m}^{2}$ of bridge plan area and production rate in $\mathrm{m}^{2} /$ week. The paper also recommends the most favourable bridge construction methods that are expected to contribute better into saving cost and construction time.
\end{abstract}




\section{Introduction}

Bridges are important components of highway networks which need to provide adequate safety and serviceability for the public. Commonly used modern bridge construction methods include Fullspan Launching Method, Advancing Shoring Method, Balanced Cantilever Method, Incremental Launching Method, and Precast Segmental Method ...etc. Wardhana and Hadipriono ${ }^{[1]}$ concluded that $12(7.6 \%)$ out of 157 bridge collapses excluding natural disasters and deterioration/obsolescence bridge failures in the United States between 1989 and 2000 were due to defective design and construction. Catastrophic bridge failures such as bridge collapses during construction incurred by the use of inappropriate construction methods can cause considerable loss in terms of time, money, damage and rework. Accordingly, selecting a desirable bridge construction technology is vital for the success of bridge projects.

Bridge superstructure construction methods involve prefabricated beams or segments which are erected on false-work, by gantry (span by span or balanced cantilever), or erected by crane. Cast in-situdecks involve post tensioned girders and slabs, balanced cantilevers, incremental launching and cast in-situ ${ }^{[2]}$.

Some of the bridge site constraints affecting the selection of construction method and working activities are: land use, staging areas, navigation constraints, hydraulic conditions, flood plain information, flood level and discharge as well as the environmental considerations.

The construction Industry Institute ${ }^{[3]}$ defined constructability as the optimum use of the construction knowledge and experience in planning, design, procurement and field operations to achieve the overall project objectives with minimum cost and construction time. Mohamed and Ismail ${ }^{[4]}$ presented an innovative method of construction that has been used in Egypt for the first time to construct $6^{\text {th }}$ October Bridge extension. They found that the unit cost decreases as the number of spans is increased for both stepping shuttering and incremental launching truss systems. Harris ${ }^{[5]}$ also reported that the 
Omdurman Islamic University Journal, 2015; 11(1): 357-375

launching truss and the stepping shuttering systems are most appropriate for long bridges in the range of $300-600 \mathrm{~m}$, where the production rate for the stepping shuttering is about $300 \mathrm{~m}^{2}$ of deck surface area per week for spans up to $40 \mathrm{~m}$.

Based on Mohamed and Ismail ${ }^{[4]}$ findings, the construction progress rate for the stepping shuttering is $14 \%$ and $48 \%$ higher than that for the incremental launching truss and the traditional formwork systems, respectively. The principal advantage of the stepping shuttering system is the saving in false-work, especially for high decks. Constructability improvements are achieved in stepping method by eliminating delays in erecting and dismantling formwork for each bridge span, and it allows for higher construction progress rates compared with

other available systems.

\section{Objectives:}

This paper attempts to document for the construction methods used for bridges' superstructure in Sudan on way to assist in achieving higher performance, on future projects, regarding cost and construction time. Hence, data of recently constructed bridges is to be analysed to view optimum cost and construction time and to conclude on the most suitable bridge construction methods that can save cost and construction time.

The paper also aims at encouraging the owners and contractors to adopt using logical procedures to guide the selection of the most appropriate bridge superstructure construction method.

3. Bridge Construction Methods commonly used in Sudan:

Construction of bridges in Sudan started since the first decade of the past century. For strategic reasons and infrastructure development, the country, since 1991, conducted outstanding expansion in the road networks in terms of length, widening of carriageways, strengthening of old bridges and construction of many new bridges.

Sudan, as other countries, has adopted advanced structural technologies indicated by application of prestress, cable supported and 
Hashim Mohamed \& Eltayeb Hassan,EltEffect of the Superstructure

Construction Method on the Cost and Duration of Bridge Projects in Sudan.

suspended structures in bridge projects. The skilled human and technical resources will strongly affect to structure safety, cost and construction time. The successes in bridge projects will be reached if the project achieved quality, cost saving and completed in optimum time schedule. Therefore, selecting an appropriate bridge construction method is essential for the success of bridge construction projects.

Many construction systems have been applied to the construction of bridges in Sudan such as: launching, balanced cantilever, cable stayed, suspended and direct erection methods involving formwork and cranes mounted on barges.

The following Tables (1) to (6) summarize the basic data regarding the main old and recently constructed bridges in Sudan.

For the listed bridges note that:

i. The mentioned bridges cross either a main river or waterway.

ii. Bored pile foundations are used for all bridges.

iii. Pile cap for most bridges is located above water level. However, some pile caps were constructed under water level using sheetpiles and dewatering techniques.

iv. Pier columns and pile caps were constructed using false formwork and cast-in-situ reinforced concrete.

v. The cost of approaches is excluded for all bridges.

vi. The contactors of the listed bridges were either from Sudan, Egypt, China, Turkey, or Italy.

In light of the above it was noticed that the cost and duration of the listed bridges is highly affected by the bridge superstructure system. 
Omdurman Islamic University Journal, 2015; 11(1): 357-375

Table (1): Khartoum State Main Bridges ${ }^{[6]}$ :

\begin{tabular}{|c|c|c|c|c|}
\hline No. & $\begin{array}{l}\text { Bridge } \\
\text { Name }\end{array}$ & $\begin{array}{c}\text { Year of } \\
\text { Completion } \\
\end{array}$ & Bridge Superstructure System & $\begin{array}{c}\text { Construction } \\
\text { Method }\end{array}$ \\
\hline \multirow{2}{*}{1} & \multirow{2}{*}{$\begin{array}{l}\text { Shambat } \\
\text { Bridge }\end{array}$} & \multirow{2}{*}{1960} & $\begin{array}{l}\text { Main spans: Prestressed Concrete (PsC) } \\
\text { double cell box girders }\end{array}$ & $\begin{array}{l}\text { Balanced } \\
\text { Cantilever }\end{array}$ \\
\hline & & & $\begin{array}{l}\text { Side spans: Reinforced Concrete (RC) } \\
\text { girder bridge. }\end{array}$ & $\begin{array}{l}\text { Launching by } \\
\text { gantry frame }\end{array}$ \\
\hline \multirow[b]{2}{*}{2} & \multirow{2}{*}{$\begin{array}{l}\text { Burri } \\
\text { Bridge }\end{array}$} & \multirow{2}{*}{1972} & Main spans: PsC double cell box girders & $\begin{array}{l}\text { Balanced } \\
\text { Cantilever }\end{array}$ \\
\hline & & & Side spans: RC girder bridge. & $\begin{array}{l}\text { Launching by } \\
\text { gantry frame }\end{array}$ \\
\hline \multirow{2}{*}{3} & \multirow{2}{*}{$\begin{array}{l}\text { Salvation } \\
\text { Bridge }\end{array}$} & \multirow{2}{*}{1999} & Main spans: cast-in-place PsC box-girders & $\begin{array}{l}\text { Balanced } \\
\text { cantilever }\end{array}$ \\
\hline & & & Side spans: PsC girders, & $\begin{array}{l}\text { Launching by } \\
\text { gantry frame }\end{array}$ \\
\hline \multirow{2}{*}{4} & \multirow{2}{*}{$\begin{array}{l}\text { Almanshia } \\
\text { Bridge }\end{array}$} & \multirow{2}{*}{2005} & Main spans: cast-in-situ PsC box-girder & $\begin{array}{l}\text { Balanced } \\
\text { cantilever }\end{array}$ \\
\hline & & & Side spans: PsC girders & $\begin{array}{l}\text { Launching by } \\
\text { gantry frame }\end{array}$ \\
\hline \multirow{2}{*}{5} & \multirow{2}{*}{$\begin{array}{l}\text { Al } \\
\text { MakNimir } \\
\text { Bridge }\end{array}$} & \multirow{2}{*}{2008} & Main spans: Steel box-girders & $\begin{array}{l}\text { Incremental } \\
\text { launching. }\end{array}$ \\
\hline & & & $\begin{array}{l}\text { Middle two spans: Stayed by cables to } \\
\text { steel box pylons. }\end{array}$ & $\begin{array}{l}\text { Incremental } \\
\text { launching. }\end{array}$ \\
\hline \multirow[t]{2}{*}{6} & \multirow[t]{2}{*}{$\begin{array}{l}\text { Tuti- } \\
\text { Khartoum } \\
\text { Suspension } \\
\text { Bridge }\end{array}$} & \multirow[t]{2}{*}{2009} & $\begin{array}{l}\text { Two towers: cast in-situ RC. } \\
\text { Main span cables. } \\
\text { Deck of composite sections. }\end{array}$ & $\begin{array}{l}\text { Cables erected } \\
\text { by catwalk. } \\
\text { Deck by crane } \\
\text { and barge }\end{array}$ \\
\hline & & & Side spans: composite steel I-girders & Erected by crane \\
\hline 7 & $\begin{array}{l}\text { Al Halfaya } \\
\text { Bridge }\end{array}$ & 2010 & Spans: Composite steel I-girders sections & $\begin{array}{l}\text { Lifted by crane. } \\
\text { Deck cast-in-situ } \\
\text { RC. }\end{array}$ \\
\hline 8 & $\begin{array}{l}\text { Khor } \\
\text { Samaha } \\
\text { Bridge } \\
\end{array}$ & 2011 & Pre-cast RC I-girders & Erected by crane \\
\hline 9 & $\begin{array}{l}\text { Al Dabasin } \\
\text { Bridge }\end{array}$ & $\begin{array}{l}\text { construction } \\
\text { is on-going }\end{array}$ & Composite steel box and I-girders. & Erected by crane. \\
\hline 10 & $\begin{array}{l}\text { Tuti-Bahry } \\
\text { Bridge }\end{array}$ & $\begin{array}{l}\text { construction } \\
\text { is on-going }\end{array}$ & Cable-stayed & $\begin{array}{l}\text { Balanced } \\
\text { Cantilever } \\
\text { Method. }\end{array}$ \\
\hline
\end{tabular}


Hashim Mohamed \& Eltayeb Hassan,EltEffect of the Superstructure

Construction Method on the Cost and Duration of Bridge Projects in Sudan.

Table (2): Geometric and Construction Data of Khartoum State Main Bridges ${ }^{[6]}$ :

\begin{tabular}{|c|l|c|c|c|c|c|}
\hline No. & \multicolumn{1}{|c|}{ Bridge Name } & $\begin{array}{c}\text { Length } \\
(\mathrm{m})\end{array}$ & $\begin{array}{c}\text { Width } \\
(\mathrm{m})\end{array}$ & $\begin{array}{c}\text { Construction } \\
\text { Time } \\
(\mathrm{months})\end{array}$ & $\begin{array}{c}\text { Total Cost } \\
(\mathrm{US} \$)\end{array}$ & $\begin{array}{c}\text { Cost/m } \\
(\mathrm{US} \$)\end{array}$ \\
\hline 1 & Shambat Bridge & 1057 & 22.6 & 30 & $5,219,000$ & 218 \\
\hline 2 & Burri Bridge & 767 & 23.0 & 26 & $10,032,000$ & 569 \\
\hline 3 & Salvation Bridge & 757 & 22.7 & 24 & $32,000,000$ & 1,857 \\
\hline 4 & Alamnshia Bridge & 340 & 20.5 & 24 & $13,000,000$ & 1,784 \\
\hline 5 & $\begin{array}{l}\text { Al MakNimir } \\
\text { Bridge }\end{array}$ & 642 & 22.0 & 18 & $23,325,200$ & 1,652 \\
\hline 6 & $\begin{array}{l}\text { Tuti-Khartoum } \\
\text { Bridge }\end{array}$ & 310 & 20.0 & 18 & $16,500,000$ & 2,661 \\
\hline 7 & $\begin{array}{l}\text { Al Halfaya } \\
\text { Bridge }\end{array}$ & 910 & 27.0 & 20 & $40,000,000$ & 1,628 \\
\hline 8 & $\begin{array}{l}\text { Khor Samaha } \\
\text { Bridge }\end{array}$ & 90 & 27.5 & 3 & $03,931,200$ & 1,588 \\
\hline 9 & $\begin{array}{l}\text { Al Dabasin } \\
\text { Bridge }\end{array}$ & 1670 & 18.5 & 24 & $33,989,000$ & 1,100 \\
\hline 10 & $\begin{array}{l}\text { Tuti-Bahry } \\
\text { Bridge }\end{array}$ & 600 & 24.0 & 36 & $41,500,000$ & 2,882 \\
\hline
\end{tabular}


Table (3): Main Bridges in other States of Sudan ${ }^{[6]}$ :

\begin{tabular}{|c|c|c|c|c|}
\hline No & $\begin{array}{l}\text { Bridge } \\
\text { Name }\end{array}$ & $\begin{array}{c}\text { Year of } \\
\text { Completio } \\
\mathrm{n}\end{array}$ & $\begin{array}{c}\text { Bridge Superstructure } \\
\text { System }\end{array}$ & $\begin{array}{l}\text { Construction } \\
\text { Method }\end{array}$ \\
\hline 1 & $\begin{array}{l}\text { Atbara } \\
\text { Bridge }\end{array}$ & 2004 & Pre-cast RC I-girders & $\begin{array}{l}\text { Erected by } \\
\text { Crane }\end{array}$ \\
\hline \multirow{2}{*}{2} & \multirow{2}{*}{$\begin{array}{l}\text { Merow } \\
\text { e } \\
\text { Bridge }\end{array}$} & \multirow{2}{*}{2007} & 6 spans, PsC T- girders & $\begin{array}{l}\text { Launched by } \\
\text { gantry }\end{array}$ \\
\hline & & & $\begin{array}{l}3 \text { spans PsC Box- girders } \\
\text { cast insitu }\end{array}$ & $\begin{array}{l}\text { Balanced } \\
\text { cantilever }\end{array}$ \\
\hline 3 & $\begin{array}{l}\text { Shendi } \\
\text { Bridge }\end{array}$ & 2008 & $\begin{array}{l}17 \text { spans, Precast T- } \\
\text { girders, and Box-girders. }\end{array}$ & $\begin{array}{l}\text { Lifted by wires } \\
\text { from barge. }\end{array}$ \\
\hline 4 & $\begin{array}{l}\text { Aldame } \\
\mathrm{r} \\
\text { Bridge }\end{array}$ & 2009 & 40m long PsC T-girders & $\begin{array}{l}\text { Moved on rails } \\
\text { and launched by } \\
\text { gantry }\end{array}$ \\
\hline 5 & $\begin{array}{l}\text { Dongol } \\
\text { a } \\
\text { Bridge }\end{array}$ & 2009 & 40m long PsC T-girders & $\begin{array}{l}\text { moved on rails } \\
\text { and launched by } \\
\text { gantry }\end{array}$ \\
\hline 6 & $\begin{array}{l}\text { Al } \\
\text { Dabba } \\
\text { Bridge }\end{array}$ & 2010 & 40m long PsC T-girders & $\begin{array}{l}\text { moved on rails } \\
\text { and launched by } \\
\text { gantry }\end{array}$ \\
\hline \multirow{2}{*}{7} & \multirow{2}{*}{$\begin{array}{l}\text { Rufaa } \\
\text { Bridge }\end{array}$} & \multirow{2}{*}{2010} & $\begin{array}{l}\text { Main spans: cast in-situ } \\
\text { concrete box girder, }\end{array}$ & $\begin{array}{l}\text { Balanced } \\
\text { cantilever }\end{array}$ \\
\hline & & & $\begin{array}{l}\text { Approach spans: cast in } \\
\text { place }\end{array}$ & By formwork \\
\hline \multirow{2}{*}{8} & \multirow{2}{*}{$\begin{array}{l}\text { Alduei } \\
\mathrm{m} \\
\text { Bridge }\end{array}$} & \multirow{2}{*}{2011} & $\begin{array}{l}\text { Main spans: cast in-situ } \\
\text { concrete box girder, }\end{array}$ & $\begin{array}{l}\text { Balanced } \\
\text { cantilever }\end{array}$ \\
\hline & & & $\begin{array}{l}\text { Approach spans: cast in } \\
\text { place }\end{array}$ & By formwork \\
\hline 9 & $\begin{array}{l}\text { Sinnar } \\
\text { Bridge }\end{array}$ & $\begin{array}{l}\text { Constructi } \\
\text { on is on- } \\
\text { going }\end{array}$ & PsC box-girders. & $\begin{array}{l}\text { Balanced } \\
\text { Cantilever }\end{array}$ \\
\hline
\end{tabular}


Hashim Mohamed \& Eltayeb Hassan,EltEffect of the Superstructure

Construction Method on the Cost and Duration of Bridge Projects in Sudan.

Table (4): Geometric and Construction Data of Main Bridges in other States of Sudan ${ }^{[6]}$ :

\begin{tabular}{|c|c|c|c|c|c|c|}
\hline No. & Bridge Name & $\begin{array}{l}\text { Length } \\
\text { (m) }\end{array}$ & $\begin{array}{l}\text { Width } \\
\text { (m) }\end{array}$ & $\begin{array}{c}\text { Construction } \\
\text { Time } \\
\text { (months) }\end{array}$ & $\begin{array}{l}\text { Total Cost } \\
\text { (US\$) }\end{array}$ & $\begin{array}{c}\operatorname{Cost} / \mathrm{m}^{2} \\
(\mathrm{US} \$)\end{array}$ \\
\hline 1 & $\begin{array}{l}\text { Atbara } \\
\text { Bridge }\end{array}$ & 440 & 18 & 15 & $12,650,000$ & 1,597 \\
\hline 2 & $\begin{array}{l}\text { Merowe } \\
\text { Bridge }\end{array}$ & 396 & 20.5 & 30 & $13,000,000$ & 1,601 \\
\hline 3 & $\begin{array}{l}\text { Shendi } \\
\text { Bridge }\end{array}$ & 660 & 20.5 & 27 & $24,400,000$ & 1,803 \\
\hline 4 & $\begin{array}{l}\text { Aldamer } \\
\text { Bridge }\end{array}$ & 858 & 20.5 & 24 & $27,600,000$ & 1,569 \\
\hline 5 & $\begin{array}{l}\text { Dongola } \\
\text { Bridge }\end{array}$ & 694 & 20.6 & 24 & $23,700,000$ & 1,628 \\
\hline 6 & $\begin{array}{l}\text { Al Dabba } \\
\text { Bridge }\end{array}$ & 366 & 20.6 & 14 & $18,000,000$ & 2,399 \\
\hline 7 & Rufaa Bridge & 354 & 11 & 24 & $13,000,000$ & 3,338 \\
\hline 8 & $\begin{array}{l}\text { Alddueim } \\
\text { Bridge }\end{array}$ & 990 & 11 & 32 & $34,000,000$ & 3,122 \\
\hline $9 a$ & $\begin{array}{l}\text { Sinnar } \\
\text { Highway } \\
\text { Bridge } \\
\end{array}$ & 310 & 18.4 & \multirow{2}{*}{36} & $24,675,824$ & 4,326 \\
\hline $9 b$ & $\begin{array}{l}\text { Sinnar } \\
\text { Railway } \\
\text { Bridge } \\
\end{array}$ & 450 & 7 & & $21,430,975$ & 6,803 \\
\hline
\end{tabular}

4. Comparison Regarding Cost and Production Rate:

Based on the above mentioned bridge data, comparison can be made using the construction cost and duration. Data of the main bridges constructed in Sudan during the period 1999 to 2011 incorporated with their construction methods, duration and the cost per square meter and of production rate are summarized in Table (5).

In Table (5), note that the production per week is a "comprehensive measure" calculated as follows:

Production rate $=($ Length of bridge $\times$ its width $) /$ construction time in weeks. 
Omdurman Islamic University Journal, 2015; 11(1): 357-375

Table (5): Cost and Production Rate

\begin{tabular}{|c|l|c|l|c|c|}
\hline No. & Bridge Name & $\begin{array}{c}\text { Year of } \\
\text { Completio } \\
\mathrm{n}\end{array}$ & $\begin{array}{l}\text { Construction } \\
\text { Method }\end{array}$ & $\begin{array}{c}\text { Cost/m } \\
\text { (US\$) }\end{array}$ & $\begin{array}{c}\text { Production } \\
\text { Rate } \\
\left(\mathrm{m}^{2} / \text { week }\right)\end{array}$ \\
\hline 1 & $\begin{array}{l}\text { Salvation } \\
\text { Bridge }\end{array}$ & 1999 & $\begin{array}{l}\text { Balanced } \\
\text { Cantilever }\end{array}$ & 1,857 & 167.1 \\
\hline 2 & $\begin{array}{l}\text { Atbara } \\
\text { Bridge }\end{array}$ & 2004 & $\begin{array}{l}\text { Erected by } \\
\text { Crane }\end{array}$ & 1,597 & 123.2 \\
\hline 3 & $\begin{array}{l}\text { Alamnshia } \\
\text { Bridge }\end{array}$ & 2005 & $\begin{array}{l}\text { Balanced } \\
\text { Cantilever }\end{array}$ & 1,784 & 67.8 \\
\hline 4 & $\begin{array}{l}\text { Merowe } \\
\text { Bridge }\end{array}$ & 2007 & $\begin{array}{l}\text { Launched by } \\
\text { gantry }\end{array}$ & 1,601 & 63.1 \\
\hline 5 & $\begin{array}{l}\text { Shendi } \\
\text { Bridge }\end{array}$ & 2008 & $\begin{array}{l}\text { Lifted by } \\
\text { wires from } \\
\text { barge }\end{array}$ & 1,803 & 116.9 \\
\hline 6 & $\begin{array}{l}\text { Al MakNimir } \\
\text { Bridge }\end{array}$ & 2008 & $\begin{array}{l}\text { Incremental } \\
\text { launching. }\end{array}$ & 1,652 & 183.1 \\
\hline 7 & $\begin{array}{l}\text { Tuti- } \\
\text { Khartoum } \\
\text { Bridge }\end{array}$ & 2009 & $\begin{array}{l}\text { Crane and } \\
\text { barge }\end{array}$ & 2,661 & 80.4 \\
\hline 8 & $\begin{array}{l}\text { Aldamer } \\
\text { Bridge }\end{array}$ & 2009 & $\begin{array}{l}\text { Launched by } \\
\text { gantry }\end{array}$ & 1,569 & 171.0 \\
\hline 9 & $\begin{array}{l}\text { Dongola } \\
\text { Bridge }\end{array}$ & 2009 & $\begin{array}{l}\text { Launched by } \\
\text { gantry }\end{array}$ & 1,628 & 139.0 \\
\hline 10 & $\begin{array}{l}\text { Al Dabba } \\
\text { Bridge }\end{array}$ & 2010 & $\begin{array}{l}\text { Launched by } \\
\text { gantry }\end{array}$ & 2,399 & 125.7 \\
\hline 11 & Rufaa Bridge & 2010 & $\begin{array}{l}\text { Balanced } \\
\text { Cantilever }\end{array}$ & 3,338 & 37.9 \\
\hline 12 & $\begin{array}{l}\text { Al Halfaya } \\
\text { Bridge }\end{array}$ & 2010 & $\begin{array}{l}\text { Erected by } \\
\text { Crane }\end{array}$ & 1,628 & 286.7 \\
\hline 13 & $\begin{array}{l}\text { Khor Samaha } \\
\text { Bridge }\end{array}$ & 2011 & $\begin{array}{l}\text { Erected by } \\
\text { Crane }\end{array}$ & 1,588 & 192.5 \\
\hline 14 & $\begin{array}{l}\text { Alddueim } \\
\text { Bridge }\end{array}$ & 2011 & $\begin{array}{l}\text { Balanced } \\
\text { Cantilever }\end{array}$ & 3,122 & 79.4 \\
\hline & & & & \\
\hline
\end{tabular}


The following notes and findings are obtained from Table (5):

i. Bridges No. 1, 6, 8, 12, and 13 show relatively less cost and high production rate.

ii. Production rate of Bridge No. 4 was slightly affected by accident during construction: flood washed-out the temporary service bridge.

iii. Bridge No. 7 (suspension bridge) is a special bridge type which is meant to be a land-mark at Khartoum city-centre, hence, the cost and duration is seriously affected.

iv. The high cost and low production rate of Bridges No. 11 and 14 is owing to the narrow width of the bridges and to the contractor being working on both bridges simultaneously.

v. Incremental Launching Method for composite bridges and Launching by gantry for prestressed concrete T-girders are acceptable construction methods for bridge superstructure.

vi. Construction by crane mounted on barge for composite steel Igirder bridge seems to be acceptable solution (Note that for Alhalfaya Bridge: $\operatorname{cost}=1,628 \mathrm{US} \$ / \mathrm{m}^{2}$; production rate $=286.7$ $\mathrm{m}^{2} /$ week).

Table (6) present the average cost and production rates for 10 of the 14 bridges; excluding Bridges No. 4, 7, 11 and 14 because of the above reasons:

Table (6): Average Cost and Production Rate:

\begin{tabular}{|l|l|c|c|}
\hline \multicolumn{1}{|c|}{ Bridge No. } & \multicolumn{1}{|c|}{$\begin{array}{c}\text { Construction } \\
\text { Method }\end{array}$} & $\begin{array}{c}\text { Average } \\
\text { Cost/m } \\
(\mathrm{US} \$)\end{array}$ & $\begin{array}{c}\text { Average } \\
\text { Production } \\
\text { Rate } \\
\left(\mathrm{m}^{2} / \mathrm{week}\right)\end{array}$ \\
\hline 1 and 3 & Balanced Cantilever & 1,821 & 117.5 \\
\hline 6 & Incremental Launching & 1,652 & 183.1 \\
\hline 8,9 and 10 & Launching by Gantry & 1,865 & 145.2 \\
\hline $2,5,12$ and 13 & $\begin{array}{l}\text { Erection by Crane or } \\
\text { Wires }\end{array}$ & 1,654 & 179.8 \\
\hline
\end{tabular}


5. Check of Application of Fuzzy Analytical Hierarchy Process (FAHP):

The Analytical Hierarchy Process (AHP) method has been widely used for solving multi-criteria decision-making problems ${ }^{[4], ~[7]}$. The Authors applied FAHP similar to Nang ${ }^{[8]}$ into local bridge project in Sudan: Cable-stayed Tuti-Bahri Bridge Project, Bridge No. 10 in Table (1).

The results were successfully applied and adopted for selection of the most appropriate bridge superstructure construction method among the two methods outlined hereafter:

i. Incremental Launching: with construction sequence as follows:

1. Construct piers of the towers.

2. Introduce temporary intermediate piers.

3. Erect deck segments by incremental launching.

4. Construct the upper part of the towers.

5. Erect the cables.

6. Remove temporary piers

ii. Balanced Cantilever: with construction sequence as follows:

1. Construct the towers.

2. Introduce cables.

3. Erect deck segments, two segments: each on one side of the towers.

The suggested procedure will be recalled here, in short, for convenience. The proposed approach employs triangular and trapezoidal fuzzy numbers and the $\alpha$-cut concept to deal with the imprecision inherent to the process of subjective judgment ${ }^{[9]}$ see Figure (1): triangle LNR and trapezoid LMOR.

In Figure (1): The vertical axis represents values of $\alpha$-cut; $L, X_{a M}$ and $R$ being the left, middle and right fuzzy numbers for the triangular $\alpha$ cut, respectively; $L, X_{a L}, X_{a R}$ and $R$ being the two left and two right fuzzy numbers for trapezoidal $\alpha$-cut. For more information see also the note under Table (7) and Reference ${ }^{[8]}$. 


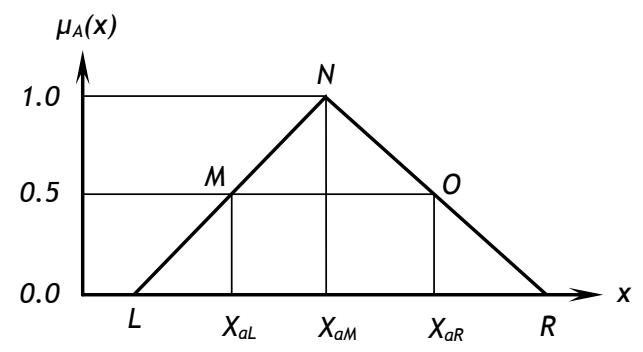

Fig. (1): Triangular and trapezoidal fuzzy intervals under $\alpha$ - cut

A case study that evaluates bridge construction methods is checked by the Authors to illustrate the use of Nang model:

i. Two expert engineers, specialized in bridge construction, were asked to identify possible factors that could affect the final decision through several survey questionnaires. The criteria used in the hierarchy were obtained and checked through the discussion process using Delphi approach ${ }^{[7]}$. Table (7) illustrates the judgments and the fuzzy numbers adopted in the process.

ii. Five main criteria for judgment on the selection of either of the two construction methods were adopted, namely: Quality, Cost, Safety, Duration, and Shape. The main criteria were further subdivided into sub-criteria as follows: Quality was characterized by durability and suitability; Cost was divided into construction cost and damage cost; Safety was associated with traffic conflict and site condition; Duration was divided into weather condition and constructability that affects productivity; Shape was divided into landscape, geometry, and environmental preservation.

iii. Once the hierarchy was established, the opinion of the two experts is used for direct pair-wise comparison and judgment. An example of experts' opinion with respect to the overall goal is shown in Tables (8) and (9). The overall goal being: "Selection of the most appropriate bridge construction method for Tuti-Bahri Bridge". 
Omdurman Islamic University Journal, 2015; 11(1): 357-375

Table (7): Fuzzy importance scale*:

\begin{tabular}{|l|c|l|l|}
\hline \multicolumn{1}{|c|}{ Judgment } & Abbreviation & \multicolumn{1}{|c|}{ Explanation } & \multicolumn{1}{|c|}{$\begin{array}{c}\text { Fuzzy } \\
\text { number }^{[8]}\end{array}$} \\
\hline $\begin{array}{l}\text { Very } \\
\text { Unimportant }\end{array}$ & VU & $\begin{array}{l}\text { A criterion is } \\
\text { strongly inferior to } \\
\text { another }\end{array}$ & $(0,0,1,2)$ \\
\hline Less Important & LI & $\begin{array}{l}\text { A criterion is slightly } \\
\text { inferior to another }\end{array}$ & $(1,2.5,4)$ \\
\hline $\begin{array}{l}\text { Equally } \\
\text { Important }\end{array}$ & $\begin{array}{l}\text { Two criteria } \\
\text { contribute equally to } \\
\text { the object }\end{array}$ & $(3,5,7)$ \\
\hline More Important & MI & $\begin{array}{l}\text { Judgment slightly } \\
\text { favor one criterion } \\
\text { over another }\end{array}$ & $(6,7.5,9)$ \\
\hline Very Important & VI & $\begin{array}{l}\text { Judgment strongly } \\
\text { favor one criterion } \\
\text { over another }\end{array}$ & $(8,9,10$, \\
$10)$
\end{tabular}

* Note that the fuzzy number for "Very Unimportant" and "Very Important" judgments are represented by half trapezoidal membership functions; whereas the remaining other 3 levels are characterized by symmetric triangular membership functions. The fuzzy numbers shown in Table (7) are suggested by Nang ${ }^{[8]}$ and accepted, after being checked by the Authors. 
Table (8): Evaluation results of the main criterion with respect to the overall goal:

\begin{tabular}{|l|c|c|}
\hline \multicolumn{1}{|c|}{ Pair-wise criteria } & $\begin{array}{c}\text { Opinion of } \\
\text { expert \#1 }\end{array}$ & $\begin{array}{c}\text { Opinion of } \\
\text { expert \#2 }\end{array}$ \\
\hline Quality versus Cost & VI & MI \\
\hline Quality versus Safety & EI & VI \\
\hline $\begin{array}{l}\text { Quality versus } \\
\text { Duration }\end{array}$ & VI & VI \\
\hline Quality versus Shape & VI & EI \\
\hline Cost versus Safety & LI & MI \\
\hline Cost versus Duration & LI & VI \\
\hline Cost versus Shape & MI & MI \\
\hline $\begin{array}{l}\text { Safety versus } \\
\text { Duration }\end{array}$ & VI & MI \\
\hline Safety versus Shape & VI & EI \\
\hline $\begin{array}{l}\text { Duration versus } \\
\text { Shape }\end{array}$ & MI & MI \\
\hline
\end{tabular}

Table (9): Evaluation results of the sub-criteria regarding the main criteria

\begin{tabular}{|l|c|c|}
\hline \multicolumn{1}{|c|}{ Pair-wise criteria } & $\begin{array}{c}\text { Opinion of } \\
\text { expert \#1 }\end{array}$ & $\begin{array}{c}\text { Opinion of } \\
\text { expert \#2 }\end{array}$ \\
\hline Durability versus Suitability & EI & VI \\
\hline Damage cost versus Construction cost & MI & MI \\
\hline Traffic conflict versus Site condition & MI & EI \\
\hline Constructability versus Weather condition & MI & VI \\
\hline Landscape versus Geometry & EI & LI \\
\hline $\begin{array}{l}\text { Landscape versus Environmental } \\
\text { preservation }\end{array}$ & LI & EI \\
\hline $\begin{array}{l}\text { Geometry versus Environmental } \\
\text { preservation }\end{array}$ & LI & MI \\
\hline
\end{tabular}


iv. The final alternative weight can be derived by summing up all the weights; the total and average weights for Incremental Launching Method and Balanced Cantilever Method are shown in the last two rows of Table (10) for $\alpha=0,0.5$ and 1.0.

From Table (10) it is clear that the Balanced Cantilever Method (weight $=0.526$ ) is more appropriate alternative than Incremental Launching Method (weight $=0.448$ ) for the adopted case study.

It is worthwhile mentioning that the Balanced Cantilever is the method adopted by the contractor and approved by the owner for the case study bridge. Construction is on-going, expected to end in 2014.

Table (10): Overall weights of the alternatives (for the two experts)

\begin{tabular}{|l|c|c|c|c|c|c|}
\hline \multirow{2}{*}{ Sub-criteria } & \multicolumn{6}{c|}{ Construction method } \\
\cline { 2 - 8 } & \multicolumn{2}{|c|}{ Incremental launching } & \multicolumn{2}{c|}{ Balanced cantilever } \\
\hline Durability & 0.0 & 0.5 & 1.0 & 0.0 & 0.5 & 1.0 \\
\hline Suitability & 0.081 & 0.080 & 0.084 & 0.134 & 0.134 & 0.135 \\
\hline Damage cost & 0.039 & 0.035 & 0.047 & 0.056 & 0.053 & 0.029 \\
\hline Construction cost & 0.037 & 0.037 & 0.037 & 0.064 & 0.063 & 0.064 \\
\hline Traffic conflict & 0.020 & 0.020 & 0.020 & 0.036 & 0.036 & 0.036 \\
\hline Site condition & 0.068 & 0.068 & 0.068 & 0.068 & 0.068 & 0.068 \\
\hline Constructability & 0.065 & 0.064 & 0.065 & 0.039 & 0.040 & 0.039 \\
\hline Weather condition & 0.065 & 0.064 & 0.057 & 0.038 & 0.035 & 0.040 \\
\hline Landscape & 0.017 & 0.017 & 0.018 & 0.023 & 0.022 & 0.024 \\
\hline Geometry & 0.017 & 0.017 & 0.017 & 0.017 & 0.017 & 0.017 \\
\hline Environmental Preservation & 0.020 & 0.021 & 0.025 & 0.028 & 0.025 & 0.028 \\
\hline Sum of Weights & 0.449 & 0.442 & 0.453 & 0.537 & 0.527 & 0.513 \\
\hline Average Weights & & 0.448 & & & 0.526 & \\
\hline
\end{tabular}




\section{Conclusions:}

The following conclusions have been drawn from this study:

i. Selection process of the superstructure construction method requires high knowledge and experience based on the information on the erection point, surrounding conditions, main characteristics of the bridge, erection machinery and material, which is usually performed based on the objective and subjective judgments of experts in the related bridge site.

ii. The Incremental Launching Method for composite bridge used in AlmakNimir Bridge and the launching system by gantry for prestressed concrete girders used in Aldamer Bridge, are acceptable and suitable methods for superstructure erection.

iii. Involving of crane and barge for erection of substructure usedin Alhalfaya Bridge can be considered as the most efficient method, and suitable for saving cost and construction time.

iv. Selection the most appropriate bridge construction method among others methods can be performed based on experts' opinion using FAHP models.

v. Using FAHP model in the selection of a most appropriate bridge construction method indicate that the balanced cantilever method is more suitable for the erection of the new Cable-stayed Bridge connecting Tuti Island to Bahri Town in Khartoum State.

vi. At normal conditions, construction cost of bridges in Sudan varies between 2,399 and 1,628 US\$/ $\mathrm{m}^{2}$ while production rate is between 286.7 and $67.8 \mathrm{~m}^{2} /$ week.

7. Definition of Important Terms (تعريف المصطلحات المهمة) 
Table (11): Definition of Terms

\begin{tabular}{|c|c|}
\hline Term(المصطلح) & Definition (التعريف) \\
\hline Balanced cantilever & كابولي منوازن \\
\hline Box-Girders & رو افد مقطعها صندوقي الثكل \\
\hline Bridge superstructure & المنشأة العلوية التي تشييد فوق دعامات الجسر \\
\hline Cable-stayed bridge & الجسور المستقرة على كو ابل \\
\hline Cast-in-situ concrete & خرسانة مصبوبة في الموقع \\
\hline Cat-walk & ممر ضيق من الحبال المعلقة \\
\hline $\begin{array}{l}\text { Fuzzy Analytical Hierarchy } \\
\text { Process }\end{array}$ & المعالجة بالتحليل الترنيبي العشوائي \\
\hline I-Girders & رو افد مقطعها يشبه الحرف I \\
\hline Incremental launching & التركيب بالتدريج: دفع بلاطة السطح في \\
\hline Prestressed concrete (PsC) & خرسانة سابقة الإجهاد \\
\hline Reinforced concrete (RC) & خرسانة مسلحة \\
\hline Span & بحر: المسافة بين دعامات الجسر \\
\hline Suspension bridge & الجسور المعلقة، مثل جسر توني_الخرطوم \\
\hline T-Girders & رو افد مقطعها يشبه الحرف T \\
\hline
\end{tabular}


Hashim Mohamed \& Eltayeb Hassan,EltEffect of the Superstructure

Construction Method on the Cost and Duration of Bridge Projects in Sudan.

\section{References:}

[1] Wardhana, K.; Hadipriono, F.C. "Analysis of recent bridge failures in the United States, ASCE" Journal of Performance of Constructed Facilities, Vol. 17 (3), pp. 144-150, 2003.

[2] Chauhan K. C. "Modern Practice on Bridge Construction on World Railways". Session No. 626, Bridges \& General (IRICE), Indian Railways Institute of Civil Engineering, India, 2010.

[3] The Construction Industry Institute, "Constructability a Primer", Publication 3-1, University of Texas, USA, 1982.

[4] Mohamed, E. A.; Ismail, M. B.: "Constructability Improvement of Bridges using Stepping Formwork", Journal of Construction Engineering and Management. May/June (2001), pp. 206-213, Alexandria, Egypt.

[5] Harries F. Modern. "Construction and Ground Engineering Equipment and Methods". Longman's, London, 1994.

[6] Ministry of Planning and Infrastructure (MPI), "Sudan Bridges Records", Khartoum State, Sudan, 2013.

[7] Buckley J. J. "Fuzzy Hierarchical Analysis", Fuzzy Sets and Systems, Vol. 17. pp. 233-247, 1985.

[8] Nang, F. P. "Fuzzy AHP Approach for Selecting the Suitable Bridge Construction Method", Automation in Construction, Vol. 17, pp. 958965, 2008.

[9] Linstone H. A.,Turoff M. "The Delphi Method, Techniques and Application", Addison-Wesley, London, 1975. 